\title{
Improving Neonatal and Maternal Outcome by Inducing Mild Labor before Elective Cesarean Section: The Lacarus Randomized Controlled Trial
}

\author{
Sven Wellmann ${ }^{\mathrm{a}, \mathrm{b}} \quad$ Gwendolin Manegold-Brauer $^{\mathrm{c}}$ Tina Fischer ${ }^{\mathrm{d}}$ \\ Leonhard Schäffer ${ }^{e}$ Vincent D. Gaertner ${ }^{f}$ Sara Fill Malfertheiner ${ }^{g}$ \\ Tilo Burkhardt ${ }^{\mathrm{h}}$
}

\begin{abstract}
aUniversity Children's Hospital Regensburg (KUNO), University of Regensburg, Regensburg, Germany; ${ }^{b}$ Division of Neonatology, University of Basel Children's Hospital (UKBB), Basel, Switzerland; 'Department of Obstetrics, University of Basel, Women's Hospital, Basel, Switzerland; dDepartment of Obstetrics, Community Hospital St. Gallen, St. Gallen, Switzerland; 'Department of Obstetrics, Community Hospital Baden, Baden, Switzerland; ${ }^{f}$ Newborn Research, Department of Neonatology, University Hospital and University of Zurich, Zurich, Switzerland; gUniversity Department of Obstetrics and Gynecology at the Hospital St. Hedwig of the Order of St. John, University of Regensburg, Regensburg, Germany; ${ }^{\text {h}}$ Department of Obstetrics, University Hospital Zurich, Zurich, Switzerland
\end{abstract}

\section{Keywords}

Arginine vasopressin · Copeptin · Elective cesarean section · Labor · Oxytocin challenge test $\cdot$ Vaginal delivery

\begin{abstract}
Background: Newborns delivered by elective cesarean section (CS) are at higher respiratory risk than those delivered vaginally or by CS proceeded by labor (secondary CS). The oxytocin challenge test (OCT) induces uterine contractions that trigger the release of fetal hormones regulating lung fluid clearance during transition from the uterine to an airbreathing environment. Objectives: The aim is to summarize current evidence and outline the Lacarus trial protocol. Methods: Literature review informed the design of a randomized placebo-controlled multicenter trial of OCT preceding elective CS in 1,450 women with a singleton pregnancy due for CS at $>35$ weeks gestation, without preceding contractions, rupture of the membranes, or antenatal steroids. OCT comprises the infusion of oxytocin $5 \mathrm{IU} / 500 \mathrm{~mL}$ Ringer lactate at a rate of $12 \mathrm{~mL} / \mathrm{h}$, doubling every $10 \mathrm{~min}$ until inducing 5 uterine contractions per 15-min interval. The
\end{abstract}

karger@karger.com www.karger.com/neo

Karger $\frac{1}{\%}$

GOPEN ACCESS
(C) 2021 The Author(s)

Published by S. Karger AG, Basel

This is an Open Access article licensed under the Creative Commons Attribution-NonCommercial-4.0 International License (CC BY-NC) (http://www.karger.com/Services/OpenAccessLicense), applicable to the online version of the article only. Usage and distribution for commercial purposes requires written permission. primary endpoint is the occurrence of neonatal respiratory morbidity within $24 \mathrm{~h}$ after birth. Secondary endpoints include biochemical and physiological parameters of fetal and maternal well-being, such as breastfeeding rate and fetal plasma copeptin concentrations. Conclusion: This is the first trial to test the hypothesis that oxytocin-induced contractions before elective CS is a promising application of physiologic principles gleaned from natural birth to improve neonatal and maternal outcomes.

(c) 2021 The Author(s)

Published by S. Karger AG, Basel

\section{Introduction}

Elective cesarean section (CS) before the onset of labor or rupture of membranes, when compared to CS or vaginal birth after the onset of labor, carries an increased risk of neonatal respiratory morbidity, including respiratory distress syndrome (RDS) or transient tachypnea of the

Clinical trial registration: www.clinicaltrials.gov, NCT03693885.
Thilo Burkhardt

Department of Obstetrics, Zurich University Hospital Frauenklinikstr. 10

$\mathrm{CH}-\mathrm{Zürich} 8091$ (Switzerland)

tilo.burkhardt@usz.ch 


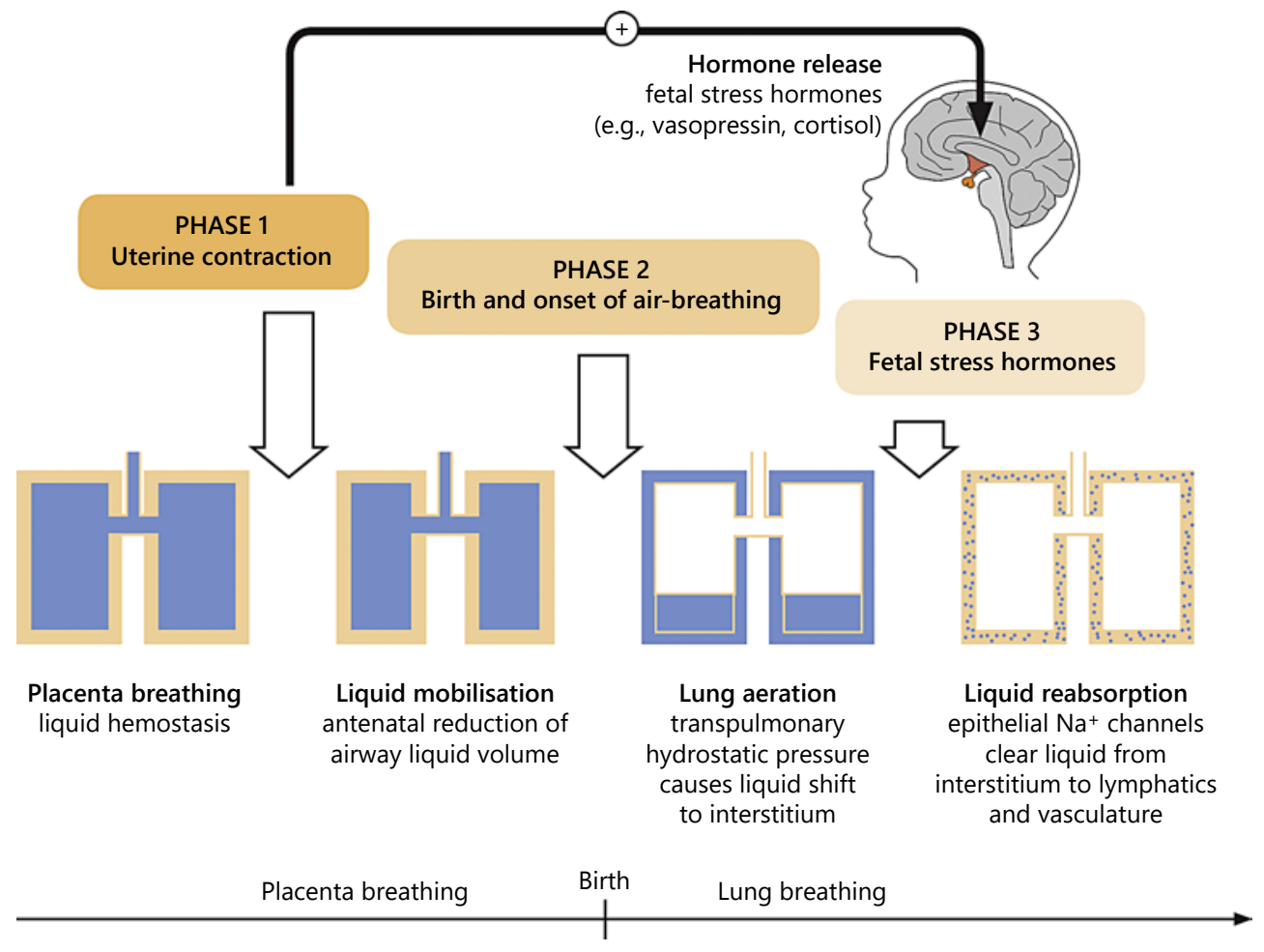

Fig. 1. Scheme of the study hypothesis.

newborn $[1,2]$. This is primarily thought to be due to an inadequate clearance of postnatal airway liquid in particular $[3,4]$. Respiratory transition at birth comprises 3 overlapping physiologic processes inextricably linked to labor (Fig. 1). First, uterine contractions not only prepare the maternal body for birth but also support the fetus by compressing its lungs, which during gestation are filled with liquid essential for lung growth that must be cleared from the airways at birth to allow air entry [5]. Uncleared liquid increases the time to aeration and causes respiratory morbidity [6]. Second, infant inspiration increases transpulmonary hydrostatic pressure gradients resulting in rapid airway liquid clearance into surrounding interstitial tissue and establishing functional residual capacity within a few minutes [7]. Third, in a final step supported by stress hormone-induced activation of epithelial $\mathrm{Na}^{+}$ channels leading to transepithelial $\mathrm{Na}^{+}$uptake [8] which concurrently leads to osmotic liquid absorption. Finally, liquid in the interstitial lung tissue is resorbed over the next hours via the lymphatics and vasculature [9].

Vaginal delivery of a healthy newborn provokes a unique surge in stress hormone plasma concentrations incommensurable with child or adult levels measured in any other situation [10]. Plasma copeptin, a nonspecific but more sensitive stress indicator than cortisol [11], leads this surge after vaginal delivery [12]. It derives from the prohormone of arginine vasopressin (AVP), is secreted in equimolar amounts to AVP from the pituitary into the circulatory system, and outperforms AVP in terms of measurability convenience in the clinical setting [13]. In contrast, newborns delivered by elective CS without preceding labor have low copeptin and AVP concentrations unless other stressors are present, including chorioamnionitis and fetal growth restriction $[10,14]$. In newborns delivered by CS after labor onset, copeptin concentrations fall between these 2 extremes [12, 15] (Fig. 1).

The oxytocin challenge test (OCT) has been a standard procedure for many decades in pregnancies with suspected placental insufficiency [16]. In a previous study, we showed that copeptin, a surrogate marker of plasma vasopressin, is markedly increased in the umbilical cord blood of infants exposed to OCT-induced contractions before elective CS [15]. 
We hypothesize that the mild uterine contractions induced by OCT suffice to upregulate various fetal stress hormones that enhance primary adaptation to an airbreathing environment. More particularly, there is evidence that AVP itself, which we measure using its surrogate marker copeptin, specifically supports lung adaptation, including alveolar fluid resorption and surfactant secretion $[8,17,18]$.

\section{Materials and Methods}

\section{Design}

The prospective randomized placebo-controlled trial in women due for elective CS compares the effect on neonatal respiratory morbidity of OCT-induced mild contractions versus standard preCS procedure.

\section{Recruitment and Setting}

Women are recruited in 4 Swiss obstetric units (University Hospital Zurich, University Hospital Basel, Kantonsspital Baden, Kantonsspital St. Gallen). The day before elective CS they are asked for their informed consent. Enrolment runs from August 2019 to January 2023.

\section{Inclusion and Noninclusion Criteria}

Inclusion Criteria

- Signed informed consent by women who understand the meaning and scope of the study.

- Singleton pregnancy $\geq 35$ weeks.

- Elective CS.

- No preceding contractions or rupture of the membranes.

- No contraindication to oxytocin.

\section{Maternal Noninclusion Criteria}

- Known or suspected unwillingness to follow the protocol.

- Substance abuse (heroin, cocaine, amphetamine, etc.)

- Placenta previa.

- Clinical signs of infection.

- Receiving treatment for arterial hypertension (any cause).

- Preeclampsia.

- Diabetes type I or II.

- Steroid therapy during pregnancy.

- Betablocker therapy at inclusion.

- Antenatal steroid administration for lung maturation.

- A history of $>1$ previous CS.

Fetal Noninclusion Criteria

- Chromosomal aberration.

- Malformation.

- Intrauterine growth retardation.

- Nonreassuring fetal heart rate pattern.

\section{Randomization}

Women are randomized 1:1 using the secuTrial ${ }^{\circledR}$ electronic data capture system to infusion of either oxytocin (OCT group) or Ringer lactate (placebo group).

\section{Sample Size}

Sample size was estimated from our earlier study [15] and the retrospective neonatal respiratory morbidity rates published by De Luca et al. [1]. According to the Swiss Federal Statistical Office, the rate of cesarean section in Switzerland was 32.1\% in 2018. A 2 -sample $z$-test for proportions computed in R (Version 3.0.3) revealed that a reduction in the probability of respiratory distress from $5.5 \%$ (control group) to 3\% (OCT group), given 750 women per group (total 1,500) and a 5\% significance level, produces a power of $67 \%$.

A sample size of 734 is needed per group to estimate the $95 \%$ confidence interval for the unknown probability of respiratory distress with precision of $0.0165(1.65 \%)$. We perform all analyses in STATA 15.0 software (Stata Corporation, College Station, TX, USA) using the Mann-Whitney test and $\chi^{2}$ test or Fisher's exact test, as appropriate, to compare groups at a $5 \%$ significance level. The trial is registered at https://clinicaltrials.gov/ under the identification code NCT03693885.

\section{Intervention Procedure}

After admission to the delivery room and placement of an intravenous line, women allocated to the OCT group receive oxytocin $5 \mathrm{IU} / 500 \mathrm{~mL}$ Ringer lactate at a rate of $12 \mathrm{~mL} / \mathrm{h}$, doubled every 10 min until it induces 5 uterine contractions per 15-min interval recorded with the cardiotocogram, at which point infusion is discontinued. The time interval between discontinuation of the OCT infusion and delivery can be between 30 and $240 \mathrm{~min}$.

Arterial cord blood samples are collected immediately after delivery into EDTA tubes and centrifuged. Plasma aliquots are stored at $-80^{\circ} \mathrm{C}$. Copeptin is measured in 3 batches using the BRAHMS KRYPTOR automated research sandwich immunoluminometric assay (B.R.A.H.M.S C-terminal pro-AVP luminescence immunoassay; B.R.A.H.M.S AG, Hennigsdorf, Germany), as previously described [19]. Infant stool and fresh breast milk samples taken by nurses and parents are stored at $-20^{\circ} \mathrm{C}$. DNA will be extracted using a commercial kit, and $16 \mathrm{~S}$ rRNA gene sequencing will be performed to characterize bacterial composition.

\section{Measurements and Outcomes}

\section{Primary Outcome}

The primary endpoint is the difference in respiratory morbidity rate between infants in the OCT and control groups characterized by one or more of the following criteria: tachypnea ( $>60$ breaths per minute), expiratory grunting, chest wall retraction, flaring of the nostrils, cyanosis, need for oxygen, or any positive pressure support present for $\geq 2 \mathrm{~h}$ within the first $48 \mathrm{~h}$ after birth. Equivalent diagnoses include RDS and transient tachypnea of the newborn.

Positive pressure support includes continuous positive airway pressure, high-flow nasal cannula, or mechanical ventilation. Neonatal death within $48 \mathrm{~h}$ after delivery is included in the composite outcome as a competing event. 


\section{Secondary Outcomes}

The following secondary outcomes will be analyzed:

- Neonatal intensive care unit admission within 48 h irrespective of diagnosis.

- Fetal stress parameters: copeptin and $\mathrm{pH}$ in arterial umbilical cord blood.

- Duration of exclusive breastfeeding $(<10$ isolated formula feedings are included).

- Duration of total breastfeeding (day of last breastfeeding).

- Postnatal weight change and maximal postnatal weight loss.

- Bilirubin measured at least once in blood or by transcutaneous bilirubinometry during the postnatal hospital stay.

- Maternal total blood loss.

- Retrospectively: antibiotic use in pregnancy (when and for how long).

- Prospectively: antibiotic use postpartum (when and which).

- Infant weight, length, and BMI to 1 year.

- Antibiotic use in pregnancy and first year of infant's life.

- Breast milk and infant stool samples collected and biobanked for end-of-study microbiome analysis.

\section{Visit Schedule}

\section{Screening}

Eligible women are asked for their informed consent at the latest on the day before elective CS or at the initial booking consultation.

\section{Day of Elective CS}

Women providing informed consent are randomized to the OCT or control group. Vital parameters (heart rate, blood pressure, and body temperature) are monitored pre-infusion in both groups.

Postnatal Ward (days 1-5 after Elective CS)

First infant stool sample, postnatal days 1-2.

Second infant stool sample, postnatal days 3-5.

Breast milk sample, postnatal days 3-5.

As recommended by the Swiss Society of Pediatrics, every child has a health booklet documenting its birth and preventive medical checkup data. We contact the parents by phone for their child's weight and length at 4 weeks and their breastfeeding status at 12 months. We also ask about antibiotic treatments (name, number of episodes, and treatment duration).

Contractions before Elective Cesarean

Section and Neonatal Outcome

\section{Enrolment, Randomization, and Allocation Concealment}

Women meeting all the inclusion criteria, including provision of informed consent, are reported to the trial center via secuTrial on the day of elective CS to receive a unique enrolment number followed by centralized block randomization using the secuTrial random number generator in a 1:1 ratio. Sequences of patient numbers are assigned to each center and kept confidential. The study medication (oxytocin or Ringer lactate) is randomly assigned to each patient number. The randomization documentation ("key list") is monitored. Treatment allocation is available to the study assistant responsible for the intervention. Neither parents, obstetricians nor neonatologists are aware of the treatment allocated.

\section{Dropout Criteria}

Oxytocin is not infused or infusion is stopped if a woman so requests, withdraws her consent and/or suffers painful contractions, or the fetal heart rate becomes abnormal.

\section{Minimizing Bias}

The effect of oxytocin is too evident for full blinding to be useful. Moreover, the study assistant in charge of the OCT must be unblinded for the oxytocin titration to achieve its target result: 5 contractions within $15 \mathrm{~min}$. In our earlier study, only $55 \%$ of women noticed the uterine contractions, none of which were painful. Not only is the neonatologist assessing the primary endpoint (respiratory morbidity) uninvolved in antenatal obstetric intervention, but study procedures and examination of the infant are separated in time and space, meaning that the primary endpoint rater is effectively blinded to the intervention.

\section{Discussion}

For over 20 years, antenatal corticosteroid (ANCS) administration in pregnancies at risk of preterm delivery ( $<34$ completed weeks of gestation) has been an important evidence-based practice for reducing RDS and mortality rates [20]. In late preterm infants (34-36 weeks), ANCS lowers neonatal respiratory morbidity irrespective of de- 
livery mode but is only effective in term infants $(\geq 37$ weeks) after elective CS, not after vaginal delivery $[21,22]$. Offsetting this benefit was an unexpected significant increase in neonatal hypoglycemia after ANCS treatment in late preterm infants. Due to the paucity of long-term safety data in late preterm and term infants, caution is needed before implementing ANCS routinely in pregnant women beyond 34 completed weeks of gestation $[23,24]$.

Oxytocin has long-standing indications in obstetrics and is approved by the FDA and EMA for antepartum and postpartum care, including the initiation or improvement of uterine contractions, where this is desirable and considered suitable, for example, for a trial of labor after prior CS (TOLAC) [25], to control postpartum hemorrhage [26].

In contrast to TOLAC where oxytocin is given in high doses with the intention to initiate or augment birth-effective labor, OCT doses are much lower as the aim is to induce mild to moderate labor sufficient to assess placental efficiency [15]. Recently, we found that copeptin is significantly increased in the umbilical cord blood of neonates exposed to OCT-induced contractions before elective CS, with no adverse effects [15]. Although mild, such contractions suffice to upregulate fetal stress hormones that may help to improve primary adaptation to an airbreathing environment [10]. In contrast to ANCS treatment, which directly alters the fetal stress hormone response and thus only influences the last phase of respiratory transition at birth, mild OCT-induced contractions have the potential to influence all 3 phases of the respiratory transition at birth in a positive and synergistic fashion (Fig. 1).

Since any disturbance of the postnatal bonding period adversely affects motherhood, including lactation and breastfeeding, all measures supporting early skin-to-skin contact and avoiding division of the mother-infant dyad are welcome [27]. Lowering the incidence of neonatal respiratory morbidity helps preserve the dyad and thus increase the likelihood of successful lactation and breast- feeding [28]. The key hormone in mammalian parenting and social development is oxytocin [29]. Where delivery occurs in the absence of labor, as in elective CS, endogenous oxytocin has limited action, thus endangering the natural development of motherhood [30]. Current CS practice includes the administration of synthetic oxytocin to all mothers immediately after delivery to control postpartum hemorrhage [26]. We conclude that given the current rates of elective CS and the excellent safety profile of OCT, the induction of mild contractions with oxytocin before elective CS may be a promising application of physiologic principles gleaned from natural birth to improve neonatal and maternal outcomes.

\section{Statement of Ethics}

This study received ethical approval from the Ethics Committees of Zurich, Northwest and Center Switzerland, and East Switzerland (ID 2018-01842). All women have given their written informed consent.

\section{Conflict of Interest Statement}

The authors report no potential conflicts of interest.

\section{Funding Sources}

This work was supported by the Family Larsson-Rosenquist Foundation, Rheinstrasse 1, 8500 Frauenfeld, Switzerland.

\section{Author Contributions}

Idea and Concept: Tilo Burkhardt and Sven Wellmann. Protocol and manuscript writing: Tilo Burkhardt, Sven Wellmann, and Gwendolin Manegold-Brauer. Implementation of the protocol in the clinics: Tilo Burkhardt, Gwendolin Manegold-Brauer, Tina Fischer, and Leo Schäffer. Final corrections and improvements: Vincent D. Gaertner and Sara Fill Malfertheiner.

\section{References}

1 De Luca R, Boulvain M, Irion O, Berner M, Pfister RE. Incidence of early neonatal mortality and morbidity after late-preterm and term cesarean delivery. Pediatrics. 2009 Jun; 123(6):e1064-71.

2 Wilmink FA, Hukkelhoven CW, Lunshof S, Mol BW, van der Post JA, Papatsonis DN. Neonatal outcome following elective cesarean section beyond 37 weeks of gestation: a 7-year retrospective analysis of a national registry. Am J Obstet Gynecol. 2010;202(3):250-8.
3 Sweet DG, Carnielli V, Greisen G, Hallman M, Ozek E, Te Pas A, et al. European consensus guidelines on the management of respiratory distress syndrome: 2019 update. Neonatology. 2019 Jun;115(4):432-50.

4 Hooper SB, Te Pas AB, Lang J, Van Vonderen JJ, Roehr CC, Kluckow M, et al. Cardiovascular transition at birth: a physiological sequence. Pediatr Res. 2015 May;77(5):608-14. 
5 Hooper SB, Harding R. Fetal lung liquid: a major determinant of the growth and functional development of the fetal lung. Clin Exp Pharmacol Physiol. 1995;22(4):235-47.

6 McGillick EV, Lee K, Yamaoka S, Te Pas AB, Crossley KJ, Wallace MJ, et al. Elevated airway liquid volumes at birth: a potential cause of transient tachypnea of the newborn. J Appl Physiol. 2017 Nov;123(5):1204-13.

7 Siew ML, Te Pas AB, Wallace MJ, Kitchen MJ, Lewis RA, Fouras A, et al. Positive end-expiratory pressure enhances development of a functional residual capacity in preterm rabbits ventilated from birth. J Appl Physiol. 2009 May;106(5):1487-93.

8 Jain L. Stress at birth and its inextricable link to the neonatal transition. Obstet Gynecol. 2016 Oct;128(4):685-7.

9 Liu J, Wang Y, Fu W, Yang CS, Huang JJ. Diagnosis of neonatal transient tachypnea and its differentiation from respiratory distress syndrome using lung ultrasound. Medicine. 2014 Dec;93(27):e197.

10 Evers KS, Wellmann S. Arginine vasopressin and copeptin in perinatology. Front Pediatr. 2016 Aug; 4:75.

11 Katan M, Morgenthaler N, Widmer I, Puder JJ, König C, Müller B, et al. Copeptin, a stable peptide derived from the vasopressin precursor, correlates with the individual stress level. Neuro Endocrinol Lett. 2008 Jun;29(3):3416.

12 Benzing J, Wellmann S, Achini F, Letzner J, Burkhardt T, Beinder E, et al. Plasma copeptin in preterm infants: a highly sensitive marker of fetal and neonatal stress. J Clin Endocrinol Metab. 2011 Jan;96(6):E982-5.

13 Bankir L, Bichet DG, Morgenthaler NG. Vasopressin: physiology, assessment and osmosensation. J Intern Med. 2017;282(4):284-97.
14 Burkhardt T, Schwabe S, Morgenthaler NG, Natalucci G, Zimmermann R, Wellmann S. Copeptin: a marker for stress reaction in fetuses with intrauterine growth restriction. Am J Obstet Gynecol. 2012;207(6):497.

15 Wellmann S, Koslowski A, Spanaus K, Zimmermann R, Burkhardt T. Fetal release of copeptin in response to maternal oxytocin administration: a randomized controlled trial. Obstet Gynecol. 2016;128(4):699-703.

16 Ray M, Freeman R, Pine S, Hesselgesser R. Clinical experience with the oxytocin challenge test. Am J Obstet Gynecol. 1972 Sep; 114(1):1-9.

17 Ross MG, Ervin G, Leake RD, Fu P, Fisher DA. Fetal lung liquid regulation by neuropeptides. Am J Obstet Gynecol. 1984 Oct;150(4): 421-5.

18 Cummings JJ, Carlton DP, Poulain FR, Fike CD, Keil LC, Bland RD. Vasopressin effects on lung liquid volume in fetal sheep. Pediatr Res. 1995 Jul;38(1):30-5.

19 Wellmann S, Benzing J, Cippà G, Admaty D, Creutzfeldt R, Mieth RA, et al. High copeptin concentrations in umbilical cord blood after vaginal delivery and birth acidosis. J Clin Endocrinol Metab. 2010 Nov;95(11):5091-6.

20 Wright LL, Horbar JD, Gunkel H, Verter J, Younes N, Andrews EB, et al. Evidence from multicenter networks on the current use and effectiveness of antenatal corticosteroids in low birth weight infants. Am J Obstet Gynecol. 1995;173(1):263-9.

21 Saccone G, Berghella V. Antenatal corticosteroids for maturity of term or near term fetuses: systematic review and meta-analysis of randomized controlled trials. BMJ. 2016;355: i5044.

22 Sotiriadis A, Makrydimas G, Papatheodorou S, Ioannidis JP, Mcgoldrick E. Corticosteroids for preventing neonatal respiratory morbidity after elective caesarean section at term. Cochrane Database Syst Rev. 2018 Aug;8(8): CD006614.
23 Groom KM. Antenatal corticosteroids after 34 weeks' gestation: do we have the evidence? Semin Fetal Neonatal Med. 2019 Jun;24(3): 189-96.

24 Skoll A, Boutin A, Bujold E, Burrows J, Crane J, Geary M, et al. No. 364-antenatal corticosteroid therapy for improving neonatal outcomes. Obstet Gynaecol Can. 2018;40(9): 1219-39.

25 Fishel Bartal M, Sibai BM, Ilan H, Fried M, Rahav R, Alexandroni $\mathrm{H}$, et al. Trial of labor after cesarean (TOLAC) in women with premature rupture of membranes*. J Matern Fetal Neonatal Med. 2020 Sep;33(17):2976-82.

26 Weeks AD, Fawcus S. Management of the third stage of labour: (for the Optimal Intrapartum Care series edited by Mercedes Bonet, Femi Oladapo and Metin Gülmezoglu). Best Pract Res Clin Obstet Gynaecol. 2020 Aug;67: 65-79.

27 Moore ER, Bergman N, Anderson GC, Medley N. Early skin-to-skin contact for mothers and their healthy newborn infants. Cochrane Database Syst Rev. 2016 Nov; 11(11): CD003519.

28 Fischer Fumeaux CJ, Denis A, Prudon MB, Plaisant F, Essomo Megnier-Mbo CM, Fernandes L, et al. Early use of mother's own raw milk, maternal satisfaction, and breastfeeding continuation in hospitalised neonates: a Prospective Cohort Study. Neonatology. 2018 Feb;113(2):131-9.

29 Rilling JK, Young LJ. The biology of mammalian parenting and its effect on offspring social development. Science. 2014 Aug;345(6198): 771-6.

30 Bell AF, Erickson EN, Carter CS. Beyond labor: the role of natural and synthetic oxytocin in the transition to motherhood. J Midwifery Womens Health. 2014;59(1):35-108. 